\title{
PATTERNS OF CAFFEINE USE IN A NEONATAL INTENSIVE CARE UNIT (NICU)
}

\author{
V. Bhatt-Mehta ${ }^{1}$, R.E. Schumacher ${ }^{2}$ \\ ${ }^{l}$ Clinical, Social and Administrative Sciences, College of Pharmacy, University of Michigan, ${ }^{2}$ Pediatrics, \\ University of Michigan, Ann Arbor, MI, USA
}

Background: Caffeine is indicated for apnea of prematurity (AOP). Reports of decreased incidence of cerebral palsy and cognitive impairment have increased its use. Recently it is widely used for other neonatal morbidities including in infants with respiratory distress syndrome (RDS) and chronic lung disease (CLD), periextubation and in postoperative apnea. No specific dosing guidelines for indications other than AOP being available.

Purpose: Determine the patterns of use of caffeine in a NICU.

Methods: Retrospective data on infants admitted between 11/2008 - 6/2010 on caffeine including demographics, caffeine dose, duration, date started, apnea, bradycardia and desaturation events and respiratory support ( mechanical ventilation (MV), CPAP duration or need for oxygen at 36 weeks corrected age ). Use was defined at RDS/CLD prevention for caffeine started before day of life (DOL) 7 (indication 1(IND1)) and AOP prevention/treatment if started after DOL 7 (IND 2).

Results: 124 infants. 56 received caffeine for IND1 and 69 for IND2. The gestational age $(28+/-2.6$ vs. $27+/-$ 2.5 weeks, $\mathrm{p}=0.03)$, MV days $(14+/-24$ vs. $30+/-32, \mathrm{p}=0.01)$ and need for oxygen at 36 weeks $(\mathrm{N}=48 / 56$ $(85 \%)$ vs. $\mathrm{N}=67 / 69(97 \%))$ were different for both groups. The loading dose at start of treatment was significantly different for the two groups $(17.2+/ 5.9 \mathrm{vs} 14.1+/ 4.9 \mathrm{mg} / \mathrm{kg}, \mathrm{p}=0.0004)$ while the maintenance doses were similar $(5.4+/-0.05$ vs. $5.1+/-1.1 \mathrm{mg} / \mathrm{kg}, \mathrm{p}=0.06)$

Conclusion: Starting caffeine in the first 7 days of life reduced the duration of MV and the need for oxygen at 36 weeks corrected age in this cohort. Loading doses were significantly different for each indication. 